\title{
Double-Hadron Leptoproduction in the Nuclear Medium
}

A. Airapetian, ${ }^{16}$ N. Akopov, ${ }^{27}$ Z. Akopov, ${ }^{27}$ M. Amarian, ${ }^{7,27}$ A. Andrus, ${ }^{15}$ E. C. Aschenauer, ${ }^{7}$ W. Augustyniak, ${ }^{26}$ R. Avakian, ${ }^{27}$ A. Avetissian, ${ }^{27}$ E. Avetissian, ${ }^{11}$ P. Bailey, ${ }^{15}$ S. Belostotski, ${ }^{19}$ N. Bianchi, ${ }^{11}$ H. P. Blok,${ }^{18,25}$ H. Böttcher, ${ }^{7}$ A. Borissov,${ }^{14}$ A. Borysenko, ${ }^{11}$ A. Brüll,${ }^{28, *}$ V. Bryzgalov,${ }^{20}$ M. Capiluppi,${ }^{10}$ G. P. Capitani,,${ }^{11}$ G. Ciullo, ${ }^{10}$ M. Contalbrigo, ${ }^{10}$ P. F. Dalpiaz, ${ }^{10}$ W. Deconinck, ${ }^{16}$ R. De Leo, ${ }^{2}$ M. Demey, ${ }^{18}$ L. De Nardo, ${ }^{6,23}$ E. De Sanctis, ${ }^{11}$ E. Devitsin, ${ }^{17}$ M. Diefenthaler, ${ }^{9}$ P. Di Nezza, ${ }^{11}$ J. Dreschler, ${ }^{18}$ M. Düren, ${ }^{13}$ M. Ehrenfried, ${ }^{9}$ A. Elalaoui-Moulay, ${ }^{1}$ G. Elbakian, ${ }^{27}$ F. Ellinghaus, ${ }^{5}$ U. Elschenbroich, ${ }^{12}$ R. Fabbri, ${ }^{18}$ A. Fantoni, ${ }^{11}$ L. Felawka, ${ }^{23}$ S. Frullani, ${ }^{22}$ A. Funel, ${ }^{11}$ G. Gapienko, ${ }^{20}$ V. Gapienko, ${ }^{20}$ F. Garibaldi, ${ }^{22}$ K. Garrow, ${ }^{23}$ G. Gavrilov, ${ }^{6,23}$ V. Gharibyan, ${ }^{27}$ F. Giordano, ${ }^{10}$ O. Grebeniouk, ${ }^{19}$ I. M. Gregor, ${ }^{7}$ K. Griffioen, ${ }^{18, \dagger}$ H. Guler,${ }^{7}$ C. Hadjidakis, ${ }^{11}$ M. Hartig, ${ }^{13}$ D. Hasch,${ }^{11}$ T. Hasegawa, ${ }^{24}$ W. H. Hesselink, ${ }^{18,25}$ A. Hillenbrand, ${ }^{9}$ M. Hoek, ${ }^{13}$ Y. Holler, ${ }^{6}$ B. Hommez, ${ }^{12}$ I. Hristova, ${ }^{7}$ G. Iarygin, ${ }^{8}$ A. Ivanilov, ${ }^{20}$ A. Izotov, ${ }^{19}$ H. E. Jackson, ${ }^{1}$ A. Jgoun, ${ }^{19}$ R. Kaiser, ${ }^{14}$ T. Keri, ${ }^{13}$ E. Kinney, ${ }^{5}$ A. Kisselev, ${ }^{5,19}$ T. Kobayashi, ${ }^{24}$ M. Kopytin, ${ }^{7}$ V. Korotkov ${ }^{20}$ V. Kozlov, ${ }^{17}$ B. Krauss, ${ }^{9}$ P. Kravchenko, ${ }^{19}$ V. G. Krivokhijine, ${ }^{8}$ L. Lagamba, ${ }^{2}$ L. Lapikás, ${ }^{18}$ P. Lenisa, ${ }^{10}$ P. Liebing, ${ }^{7}$ L. A. Linden-Levy, ${ }^{15}$ W. Lorenzon, ${ }^{16}$ J. Lu, ${ }^{23}$ S. Lu, ${ }^{13}$ B.-Q. Ma, ${ }^{3}$ B. Maiheu, ${ }^{12}$ N. C. R. Makins, ${ }^{15}$ Y. Mao, ${ }^{3}$ B. Marianski, ${ }^{26}$ H. Marukyan, ${ }^{27}$ F. Masoli, ${ }^{10}$ V. Mexner, ${ }^{18}$ N. Meyners, ${ }^{6}$ T. Michler, ${ }^{9}$ O. Mikloukho, ${ }^{19}$ C. A. Miller, ${ }^{23}$ Y. Miyachi, ${ }^{24}$ V. Muccifora, ${ }^{11}$ M. Murray, ${ }^{14}$ A. Nagaitsev, ${ }^{8}$ E. Nappi ${ }^{2}$ Y. Naryshkin, ${ }^{19}$ M. Negodaev, ${ }^{7}$ W.-D. Nowak, ${ }^{7}$ H. Ohsuga, ${ }^{24}$ A. Osborne, ${ }^{14}$ R. Perez-Benito, ${ }^{13}$ N. Pickert, ${ }^{9}$ M. Raithel,,${ }^{9}$ D. Reggiani, ${ }^{9}$ P. E. Reimer, ${ }^{1}$ A. Reischl, ${ }^{18}$ A. R. Roelon, ${ }^{11}$ C. Riedl, ${ }^{9}$ K. Rith, ${ }^{9}$ G. Rosner,${ }^{14}$ A. Rostomyan,,${ }^{6}$ L. Rubacek, ${ }^{13}$ J. Rubin, ${ }^{15}$ D. Ryckbosch, ${ }^{12}$ Y. Salomatin, ${ }^{20}$ I. Sanjiev, ${ }^{1,19}$ I. Savin, ${ }^{8}$ A. Schäfer, ${ }^{21}$ G. Schnell, ${ }^{24}$ K. P. Schüler, ${ }^{6}$ J. Seele, ${ }^{5}$ R. Seidl,,${ }^{9}$ B. Seitz, ${ }^{13}$ C. Shearer, ${ }^{14}$ T.-A. Shibata, ${ }^{24}$ V. Shutov, ${ }^{8}$ K. Sinram, ${ }^{6}$ M. Stancari, ${ }^{10}$ M. Statera, ${ }^{10}$ E. Steffens, ${ }^{9}$ J. J. M. Steijger, ${ }^{18}$ H. Stenzel, ${ }^{13}$ J. Stewart, ${ }^{7}$ F. Stinzing, ${ }^{9}$ J. Streit, ${ }^{13}$ P. Tait, ${ }^{9}$ H. Tanaka, ${ }^{24}$ S. Taroian,${ }^{27}$ B. Tchuiko, ${ }^{20}$ A. Terkulov, ${ }^{17}$ A. Trzcinski, ${ }^{26}$ M. Tytgat, ${ }^{12}$ A. Vandenbroucke, ${ }^{12}$ P. B. van der Nat, ${ }^{18}$ G. van der Steenhoven, ${ }^{18}$ Y. van Haarlem, ${ }^{12}$ D. Veretennikov, ${ }^{19}$ V. Vikhrov, ${ }^{19}$ C. Vogel, ${ }^{9}$ S. Wang, ${ }^{3}$ Y. Ye, ${ }^{4}$ Z. Ye, ${ }^{6}$ S. Yen, ${ }^{23}$ B. Zihlmann, ${ }^{12}$ and P. Zupranski ${ }^{26}$

\section{(HERMES Collaboration)}

${ }^{1}$ Physics Division, Argonne National Laboratory, Argonne, Illinois 60439-4843, USA

${ }^{2}$ Istituto Nazionale di Fisica Nucleare, Sezione di Bari, 70124 Bari, Italy

${ }^{3}$ School of Physics, Peking University, Beijing 100871, China

${ }^{4}$ Department of Modern Physics, University of Science and Technology of China, Hefei, Anhui 230026, China

${ }^{5}$ Nuclear Physics Laboratory, University of Colorado, Boulder, Colorado 80309-0390, USA

${ }^{6}$ DESY, 22603 Hamburg, Germany

${ }^{7}$ DESY, 15738 Zeuthen, Germany

${ }^{8}$ Joint Institute for Nuclear Research, 141980 Dubna, Russia

${ }^{9}$ Physikalisches Institut, Universität Erlangen-Nürnberg, 91058 Erlangen, Germany

${ }^{10}$ Istituto Nazionale di Fisica Nucleare, Sezione di Ferrara and Dipartimento di Fisica, Università di Ferrara, 44100 Ferrara, Italy

${ }^{11}$ Istituto Nazionale di Fisica Nucleare, Laboratori Nazionali di Frascati, 00044 Frascati, Italy

${ }^{12}$ Department of Subatomic and Radiation Physics, University of Gent, 9000 Gent, Belgium

${ }^{13}$ Physikalisches Institut, Universität Gießen, 35392 Gießen, Germany

${ }^{14}$ Department of Physics and Astronomy, University of Glasgow, Glasgow G12 8QQ, United Kingdom

${ }^{15}$ Department of Physics, University of Illinois, Urbana, Illinois 61801-3080, USA

${ }^{16}$ Randall Laboratory of Physics, University of Michigan, Ann Arbor, Michigan 48109-1040, USA

${ }^{17}$ Lebedev Physical Institute, 117924 Moscow, Russia

${ }^{18}$ Nationaal Instituut voor Kernfysica en Hoge-Energiefysica (NIKHEF), 1009 DB Amsterdam, The Netherlands

${ }^{19}$ Petersburg Nuclear Physics Institute, St. Petersburg, Gatchina 188350, Russia

${ }^{20}$ Institute for High Energy Physics, Protvino, Moscow Region 142281, Russia

${ }^{21}$ Institut für Theoretische Physik, Universität Regensburg, 93040 Regensburg, Germany

${ }^{22}$ Istituto Nazionale di Fisica Nucleare, Sezione Roma 1, Gruppo Sanità and Physics Laboratory, Istituto Superiore di Sanità, 00161 Roma, Italy

${ }^{23}$ TRIUMF, Vancouver, British Columbia V6T 2A3, Canada

${ }^{24}$ Department of Physics, Tokyo Institute of Technology, Tokyo 152, Japan

${ }^{25}$ Department of Physics and Astronomy, Vrije Universiteit, 1081 HV Amsterdam, The Netherlands

${ }^{26}$ Andrzej Soltan Institute for Nuclear Studies, 00-689 Warsaw, Poland

${ }^{27}$ Yerevan Physics Institute, 375036 Yerevan, Armenia

${ }^{28} 36$ Mizzen Circle, Hampton, Virginia 23664, USA 
(Received 11 October 2005; published 25 April 2006)

The first measurements of double-hadron production in deep-inelastic scattering within the nuclear medium were made with the HERMES spectrometer at DESY HERA using a $27.6 \mathrm{GeV}$ positron beam. By comparing data for deuterium, nitrogen, krypton, and xenon nuclei, the influence of the nuclear medium on the ratio of double-hadron to single-hadron yields was investigated. Nuclear effects on the additional hadron are clearly observed, but with little or no difference among nitrogen, krypton, or xenon, and with smaller magnitude than effects seen on previously measured single-hadron multiplicities. The data are compared with models based on partonic energy loss or prehadronic scattering and with a model based on a purely absorptive treatment of the final-state interactions. Thus, the double-hadron ratio provides an additional tool for studying modifications of hadronization in nuclear matter.

DOI: 10.1103/PhysRevLett.96.162301

Hadron production from a free nucleon in deep-inelastic scattering is generally described by fragmentation functions that contain nonperturbative information about parton hadronization. These functions are expected to be different for nuclear targets [1] because of several possible effects: energy loss of the propagating quarks, rescattering during the prehadronic formation process, or interactions of the final-state hadrons within the nucleus.

Despite recent accurate experimental data from singlehadron leptoproduction [2,3] and relativistic heavy-ion collisions [4-6], the underlying mechanisms in theoretical models for hadronization in the nuclear medium differ greatly [7-11]. In-medium modification of the quark fragmentation function has been described in terms of rescattering of gluons and quarks, and of energy loss due to induced gluon radiation $[7,8]$. Alternatively, colorless prehadron rescattering in the medium has been suggested [9,10] with additional effects due to $Q^{2}$ rescaling [11]. Older interpretations [1,12] based on hadronic final-state interactions require a hadron formation length smaller than the nuclear size, which is unlikely for struck quarks boosted to energies larger than a few GeV. Although models based on some of these ideas are already in conflict with data, clearly other types of data are needed to further distinguish among these interpretations.

Double-hadron leptoproduction offers an additional way to study hadronization. If partonic energy loss of the struck quark were the only mechanism involved, it would be naively expected that the attenuation effect does not depend strongly on the number of hadrons involved, and the double-hadron to single-hadron ratio for a nuclear target should be only slightly dependent on the mass number $A$. On the contrary, if final hadron absorption were the dominant process, the requirement of an additional slower subleading hadron that is more strongly absorbed would suppress the two-hadron yield from heavier nuclei $[9,13]$, so that this ratio should decrease with $A$.

Data from STAR [14] on hadron pair production as a function of azimuthal angle showed that for a fixed value of the trigger hadron's transverse momentum, the production of opposite-side hadron pairs is completely suppressed for central $\mathrm{Au}+\mathrm{Au}$ collisions due to the final-state interactions with the dense medium generated in such collisions. On the other hand, the same-side pairs exhibit jetlike
PACS numbers: 25.30.Rw, 13.60.-r, 13.87.Fh, 25.75.Gz

correlations that are similar to $p+p$ and $p+d$ collisions. These results were used in Ref. [15] to advocate the picture that jet fragmentation occurs outside the dense medium. In this model it has been shown that if hadron absorption or rescattering were responsible for the observed hadron suppression, it would likely destroy the jet structure and, in particular, the correlations between leading and subleading hadrons within the jet cone. However, the heavy-ion data cannot exclude hadronic absorption effects completely.

In this Letter the first measurement of double-hadron leptoproduction on nitrogen, krypton, and xenon relative to deuterium is presented. All charged hadrons and $\pi^{0}$ mesons are considered.

Semi-inclusive deep-inelastic scattering data are presented in terms of the ratio

$$
R_{2 h}\left(z_{2}\right)=\frac{\left(\frac{d N^{z_{1}}>0.5}{N^{z_{1}}\left(z_{2}\right) / d z_{2}}\right){ }_{A}}{\left(\frac{d N^{z_{1}}>0.5}{N^{z_{1}}\left(z_{2}\right) / d z_{2}}\right)_{D}},
$$

in which $z=E_{h} / \nu$ is the fractional hadronic energy, $E_{h}$ is the hadron energy, and $\nu$ is the virtual photon energy, all of which are evaluated in the target rest frame. The values $z_{1}$ and $z_{2}$ correspond to the leading (largest $z$ ) and subleading (second largest $z$ ) hadrons, respectively. The quantity $d N^{z_{1}>0.5}$ is the number of events with at least two detected hadrons in a bin of width $d z_{2}$ at $z_{2}$ with $z_{1}>0.5$. The quantity $N^{z_{1}>0.5}$ is the number of events with at least one detected hadron with $z_{1}>0.5$. The label $A(D)$ indicates that the term is calculated for a nuclear (deuterium) target.

The measurement was performed with the HERMES spectrometer [16] using the $27.6 \mathrm{GeV}$ positron beam stored in the HERA ring at DESY. The spectrometer consists of two identical halves located above and below the positron beam pipe. The scattered positrons and the produced hadrons were detected simultaneously within an angular acceptance of $\pm 170 \mathrm{mrad}$ horizontally and $\pm(40-140) \mathrm{mrad}$ vertically.

The nuclear targets, which were internal to the positron storage ring, consisted of polarized or unpolarized deuterium, or unpolarized high-density nitrogen, krypton, or xenon gas injected into a $40 \mathrm{~cm}$ long open-ended tubular storage cell. Target areal densities up to $1.4 \times$ $10^{16}$ nucleons $/ \mathrm{cm}^{2}$ were obtained for unpolarized gas corresponding to luminosities up to $3 \times 10^{33}$ nucleons $/\left(\mathrm{cm}^{2} \mathrm{~s}\right)$. 
The positron trigger was formed by a coincidence between the signals from three scintillator hodoscope planes, and a lead glass calorimeter where a minimum energy deposit of $3.5 \mathrm{GeV}(1.4 \mathrm{GeV})$ for unpolarized (polarized) target runs was required. The scattered positrons were identified using a transition-radiation detector, a scintillator preshower counter, and an electromagnetic calorimeter. Scattered positrons were selected by imposing constraints on the squared four-momentum of the virtual photon $Q^{2}>$ $1 \mathrm{GeV}^{2}$, on the invariant mass of the photon-nucleon system $W=\sqrt{2 M \nu+M^{2}-Q^{2}}>2 \mathrm{GeV}$, where $M$ is the nucleon mass, and on the energy fraction of the virtual photon $y=\nu / E<0.85$, where $E$ is the beam energy. The constraints on $W$ and $y$ are applied to exclude nucleon resonance excitations and to limit the magnitude of the radiative corrections, respectively. In addition, the requirement $\nu>7 \mathrm{GeV}$ was imposed to limit the kinematical correlations between $\nu$ and $z$.

Charged hadrons (i.e., $\pi, K$, and $p$ without distinction) were reconstructed for momenta above $1.4 \mathrm{GeV}$. The elec-

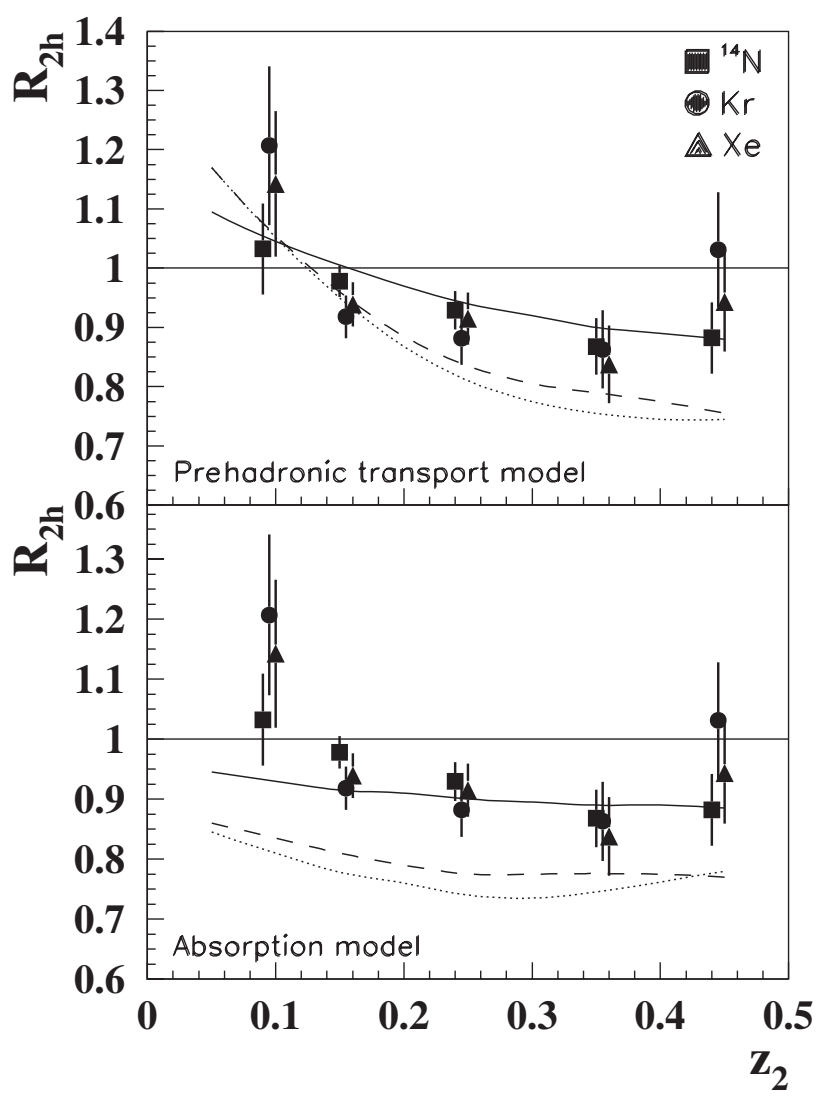

FIG. 1. The ratio $R_{2 h}$ as a function of $z_{2}$ for ${ }^{14} \mathrm{~N}$ (squares), $\mathrm{Kr}$ (circles), and Xe (triangles) with $z_{1}>0.5$. Only selection I was considered. The systematical uncertainty is $2 \%$ for all the targets and is independent of $z_{2}$. In the upper panel, the curves (solid for ${ }^{14} \mathrm{~N}$, dashed for $\mathrm{Kr}$, dotted for $\mathrm{Xe}$ ) are calculated within a BUU transport model [10]. In the bottom panel, the same data are shown with calculations that assume only absorption for the three nuclei (same line types as in the upper plot) [10]. tromagnetic calorimeter [17] provided neutral pion identification through the detection of neutral clusters originating from two-photon decay. Each of the two clusters was required to have an energy $E_{\gamma} \geq 0.8 \mathrm{GeV}$. The $\pi^{0}$ mesons were selected by requiring that the reconstructed invariant mass was within 2 standard deviations of the center of the $\pi^{0}$ mass peak.

The leading hadron was selected with $z_{1}>0.5$. In this case, it is expected to contain the struck current quark with high probability. No explicit constraint was applied to $z_{2}$. Both $z_{1}$ and $z_{2}$ were calculated assuming that all hadrons have the mass of the pion.

Using the code of Ref. [18], radiative corrections to $R_{2 h}$ were found to be negligible in the whole kinematic range. This is because there is no elastic or quasielastic tail in semi-inclusive events, and the inelastic corrections largely cancel in the measured ratio.

Two methods of double-hadron event selection were used. Selection I contains only the combinations of hadron charges (leading-subleading) $++,--,+0,0+,-0$, $0-, 00$. This suppresses the contributions from $\rho^{0} \rightarrow$ $\pi^{+} \pi^{-}$decay because the +- and -+ combinations are missing. Moreover, in the Lund string model, the exclusion of the opposite-charge combinations enhances the rank-1 (leading) plus rank-3 (subleading) combination [19]. The higher the particle rank, the more likely it is formed deep inside the nucleus, and the corresponding hadron absorption should be larger. Selection II contains all particle charge combinations. Here, the subleading hadron is mainly of rank 2 and the contribution from $\rho^{0}$ decay is larger. In both selections I and II the relative yield from exclusive $\rho^{0}$ production in $N^{z_{1}>0.5}$ is small and was evalu-

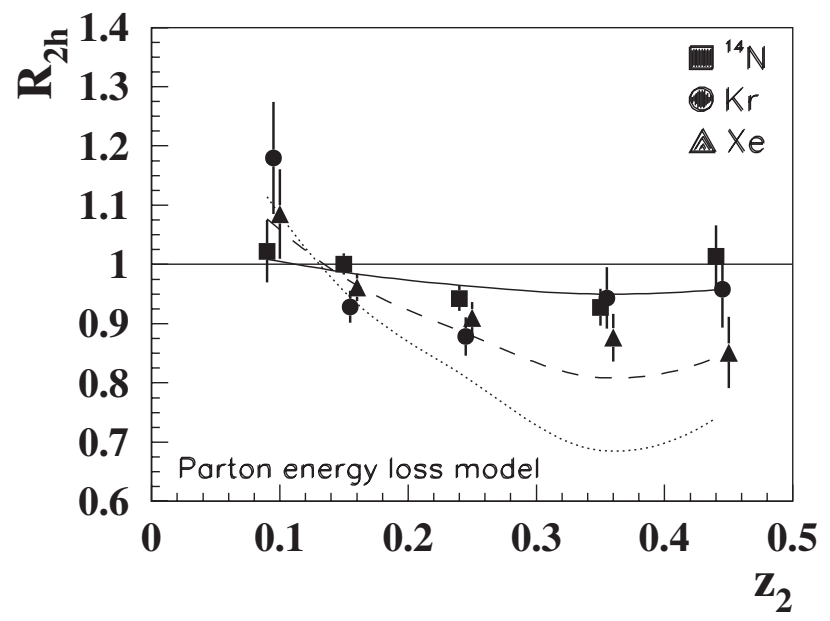

FIG. 2. The ratio $R_{2 h}$ as a function of $z_{2}$ for ${ }^{14} \mathrm{~N}$ (squares), $\mathrm{Kr}$ (circles), and Xe (triangles) with $z_{1}>0.5$ for selection II. The systematic uncertainty is $4 \%(3 \%)$ for xenon and krypton (nitrogen) and is independent of $z_{2}$. The curves $\left({ }^{14} \mathrm{~N}\right.$, solid; $\mathrm{Kr}$, dashed; $\mathrm{Xe}$, dotted) are from the parton energy loss model described in Refs. [15,22]. 
TABLE I. Averaged values of $R_{2 h}$ for $z_{1}>0.5$.

\begin{tabular}{|c|c|c|c|c|c|}
\hline & $z_{2}<0.5$ & $0.1<z_{2}<0.5$ & $\begin{array}{c}0.1<z_{2}<0.5 \\
\text { model }[10]\end{array}$ & $\begin{array}{c}0.1<z_{2}<0.5 \\
\text { model absorbtion [10] }\end{array}$ & $\begin{array}{l}0.1<z_{2}<0.5 \\
\text { model }[15,22]\end{array}$ \\
\hline${ }^{14} \mathrm{~N} / \mathrm{D}$ selection I & $0.946 \pm 0.017 \pm 0.019$ & $0.941 \pm 0.018 \pm 0.019$ & 0.931 & 0.907 & \\
\hline${ }^{14} \mathrm{~N} / \mathrm{D}$ selection II & $0.975 \pm 0.009 \pm 0.029$ & $0.972 \pm 0.010 \pm 0.029$ & & & 0.965 \\
\hline $\mathrm{Kr} / \mathrm{D}$ selection I & $0.929 \pm 0.015 \pm 0.019$ & $0.917 \pm 0.016 \pm 0.018$ & 0.835 & 0.796 & \\
\hline $\mathrm{Kr} / \mathrm{D}$ selection II & $0.902 \pm 0.008 \pm 0.036$ & $0.892 \pm 0.008 \pm 0.036$ & & & 0.879 \\
\hline $\mathrm{Xe} / \mathrm{D}$ selection I & $0.936 \pm 0.023 \pm 0.019$ & $0.915 \pm 0.024 \pm 0.018$ & 0.815 & 0.773 & \\
\hline $\mathrm{Xe} / \mathrm{D}$ selection II & $0.936 \pm 0.012 \pm 0.037$ & $0.925 \pm 0.013 \pm 0.037$ & & & 0.800 \\
\hline
\end{tabular}

ated by Monte Carlo calculation to be on the order of $12 \%$ for the deuterium target.

Figure 1 shows the double ratio $R_{2 h}$ as a function of $z_{2}$ for selection I only. The kinematic variables are in the range $\langle\nu\rangle=21$ to $16 \mathrm{GeV}$ and $\left\langle Q^{2}\right\rangle=2.1$ to $2.6 \mathrm{GeV}^{2}$ as $z_{2}$ goes from 0.09 to 0.44 . The averages over $z_{2}$ are $\langle\nu\rangle=$ $17.7 \mathrm{GeV}$ and $\left\langle Q^{2}\right\rangle=2.4 \mathrm{GeV}^{2}$.

The ratio $R_{2 h}$ is generally below unity with no significant difference between the three nuclei. These data clearly show that the nuclear effect in the double-hadron ratio is much smaller than for the single-hadron attenuation measured under the same kinematic conditions [2,3]. For $z_{2}<$ 0.1 , where $R_{2 h}$ rises towards and possibly above 1 , the slow hadrons originate largely from target fragmentation $[10,20]$. Also for $z_{2}>0.4$, where the two hadrons have similar energy, $R_{2 h}$ seems to rise towards unity.

Figure 1 (upper panel) shows calculations based on a PYTHIA event generator with a fully coupled-channel treatment of final-state interactions by means of a semiclassical Boltzmann-Uehling-Uhlenbeck (BUU) transport model [10]. In this model, the fragmentation function is modified by prehadron interactions and rescattering in the medium. Although the general trend of the data is reproduced, the model predicts an effect twice as large for xenon and krypton as for nitrogen above $z_{2}=0.1$, which is not supported by the data.

Figure 1 (bottom panel) shows the same data compared to a calculation with a purely absorptive treatment of the interaction of the prehadronic or the final hadronic states. The data rule out this assumption [10].

Figure 2 presents $R_{2 h}$ calculated for all hadron charge combinations (selection II). Inclusion of the +- and -+ pairs does not change the value of $R_{2 h}$ significantly, contrary to all of the naive space-time evolutionary models of hadronization. In order to evaluate further the possible influence of the exclusive and semi-inclusive $\rho^{0}$ production, $R_{2 h}$ was extracted for all hadron pairs except those with invariant mass near the $\rho^{0}$. This has no noticeable effect on $R_{2 h}$. Therefore, the final data are presented over the full invariant mass range. The effect of only the exclusive $\rho^{0}$ production is even smaller since it contributes only $5 \%$ of the total yield. The contamination from exclusive production of $\rho^{ \pm}$and $\omega$ mesons is completely negligible, being suppressed by more than 1 order of magnitude with respect to the $\rho^{0}$ [21].

The curves in Fig. 2 represent the model $[15,22]$ in which modifications of the fragmentation functions arises from parton energy loss. Contrary to naive expectations, this model predicts a significant $A$ dependence, in conflict with the data.

Table I provides a quantitative comparison between the data and theoretical predictions for $R_{2 h}$ integrated over $z_{2}$.

The total systematic uncertainty on $R_{2 h}$ is $4 \%$ (3\%) for xenon and krypton (nitrogen) and is nearly independent of $z_{2}$. The main contribution to the systematic uncertainty comes from the decay of exclusively produced $\rho^{0}$ mesons. However, for the double-hadron multiplicities $d N^{z_{1}>0.5}\left(z_{2}\right) / d z_{2}$, the $\rho^{0}$ contribution has a negligible effect. The $\rho^{0}$ contribution to $N^{z_{1}>0.5}$ was estimated in analogy with Refs. [2,3] to be about $2 \%$ (3\%) for light (heavy) nuclei. The only other contributing factor is the uncertainty in the overall efficiency of $2 \%$. The geometric acceptance for semi-inclusive hadron production was verified to be the same for both the nuclear and deuterium targets by studying the multiplicity ratio as a function of the hadron polar angle. This ratio is constant within experimental error.

In conclusion, the first measurement of double-hadron production on deuterium, nitrogen, krypton, and xenon is presented. The ratio of double- to single-hadron yields from nuclear targets compared to deuterium are similar for atomic mass numbers $A=14,84$, and 131 , as a function of the relative energy of the subleading hadron. This is at variance with the single-hadron attenuation data, which depend strongly on $A$. The data do not support naive expectations for prehadronic and hadronic final-state interactions that are purely absorptive. Models that interpret modifications to fragmentation as being due to prehadronic scattering or partonic energy loss are also inconsistent with the data. In fact, the latter predict an even larger $A$ dependence, while the data show little. Like the jet correlation measurements in heavy-ion collisions, the double-hadron observables in semi-inclusive deep-inelastic scattering provide new information for differentiating between models of hadronization in nuclei that are indistinguishable in singlehadron measurements. 
We are grateful to T. Falter, B. Kopeliovich, A. Majumder, and X. N. Wang for useful discussions. We gratefully acknowledge the DESY management for its support, the staff at DESY and the collaborating institutions for their significant effort, and our national funding agencies and the EU RII3-CT-2004-506078 program for financial support.

*Permanent address: Thomas Jefferson National Accelerator Facility, Newport News, VA 23606, USA.

'Permanent address: College of William \& Mary, Williamsburg, VA 23187, USA.

[1] M. Gyulassy and M. Plumer, Nucl. Phys. B346, 1 (1990); J. Czyzewski, Phys. Rev. C 43, 2426 (1991).

[2] A. Airapetian et al. (HERMES Collaboration), Phys. Lett. B 577, 37 (2003).

[3] A. Airapetian et al. (HERMES Collaboration), Eur. Phys. J. C 20, 479 (2001).

[4] C. Adler et al. (STAR Collaboration), Phys. Rev. Lett. 90, 032301 (2003); 90, 082302 (2003); J. Adams et al. (STAR Collaboration), Phys. Rev. Lett. 91, 072304 (2003); Phys. Rev. C 70, 044901 (2004); Phys. Rev. Lett. 95, 152301 (2005).

[5] K. Adcox et al. (PHENIX Collaboration), Phys. Lett. B 561, 82 (2003); S. S. Adler et al. (PHENIX Collaboration), Phys. Rev. Lett. 91, 072303 (2003); Phys. Rev. C 69, 034910 (2004); Phys. Rev. Lett. 93, 202002 (2004); K. Adcox et al. (PHENIX Collaboration), Nucl. Phys. A757, 184 (2005).

[6] B. B. Back et al. (PHOBOS Collaboration), Phys. Rev. Lett. 91, 072302 (2003); Phys. Rev. C 70, 011901 (2004); Phys. Rev. Lett. 94, 082304 (2005).
[7] X. N. Wang and X. Guo, Nucl. Phys. A696, 788 (2001); E. Wang and X.N. Wang, Phys. Rev. Lett. 89, 162301 (2002).

[8] F. Arleo, J. High Energy Phys. 11 (2002) 44; Nucl. Phys. A715, 899 (2003).

[9] B.Z. Kopeliovich, J. Nemchik, E. Predazzi, and A. Hayashigaki, Nucl. Phys. A740, 211 (2004).

[10] T. Falter et al., Phys. Lett. B 594, 61 (2004); Phys. Rev. C 70, 054609 (2004); K. Gallmeister and W. Cassing, Nucl. Phys. A748, 241 (2005).

[11] A. Accardi, V. Muccifora, and H. J. Pirner, Nucl. Phys. A720, 131 (2003).

[12] A. Bialas, Acta Phys. Pol. B 11, 475 (1980).

[13] X. N. Wang, Phys. Lett. B 595, 165 (2004); B. W. Zhang et al., Phys. Rev. Lett. 93, 072301 (2004); A. Majumder and X. N. Wang, Phys. Rev. D 70, 014007 (2004); X. N. Wang, Nucl. Phys. A750, 98 (2005).

[14] J. Adams et al. (STAR Collaboration), Phys. Rev. Lett. 91, 072304 (2003).

[15] X. N. Wang, Phys. Lett. B 579, 299 (2004).

[16] K. Ackerstaff et al. (HERMES Collaboration), Nucl. Instrum. Methods Phys. Res., Sect. A 417, 230 (1998).

[17] H. Avakian et al., Nucl. Instrum. Methods Phys. Res., Sect. A 417, 69 (1998).

[18] A. A. Akhundov, D. Yu Bardin, and N. M. Shumeiko, Sov. J. Nucl. Phys. 26, 660 (1977); D. Yu. Bardin and N. M. Shumeiko, Sov. J. Nucl. Phys. 29, 499 (1979); A. A. Akhundov et al., Sov. J. Nucl. Phys. 44, 988 (1986).

[19] T. Sjostrand et al., Comput. Phys. Commun. 135, 238 (2001).

[20] K. Gallmeister and T. Falter, Phys. Lett. B 630, 40 (2005); (private communication).

[21] M. Diehl et al., Phys. Rev. D 72, 034034 (2005); 72, 059902 (2005).

[22] A. Majumder, Eur. Phys. J. C 43, 259 (2005); (private communication). 